\title{
Decomposição e análise dos potenciais evocados auditivos de tronco encefálico
}

\author{
Kheline Fernandes Peres Naves*, Adriano Alves Pereira, Adriano Oliveira Andrade
}

Resumo Os PEATEs são sinais resultantes da combinação de respostas de atividades neurais a estímulos sonoros no córtex. Caracteriza-se por ondas, sendo seus picos nomeados por algarismos romanos (I, II, III, IV, V, VI e VII). O processo clássico de identificação desses picos é baseado na visualização do sinal gerado pela promediação de cada amostra. Nele são identificadas as características morfológicas do sinal e os aspectos temporais relevantes constituídos pelas ondas de Jewett no qual cada onda tem uma relação anatômica com o sítio de origem. No entanto, durante esse processo de identificação visual surgem dificuldades que tornam a análise visual dos PEATE uma fonte constante de dúvidas em relação a fidedignidade e concordância de marcação dos picos pela subjetividade entre os examinadores. Com o objetivo de melhorar o processo de avaliação dos PEATE, foi desenvolvido um sistema de detecção automática para os picos, com capacidade de aprendizado que leva em consideração o perfil de marcação prévia realizado por examinadores, podendo ser considerado também, as marcações futuras de examinadores que utilizarão o software como auxílio em suas análises. Para a deteç̧ão de picos foi utilizada a Transformada Wavelet Contínua, associada a um Classificador Probabilístico construído a partir de marcações realizadas pelos examinadores. Para a avaliação do sistema foram utilizadas 748 amostras de PEATE de 11 sujeitos. A avaliação do sistema proposto apresentou uma taxa de acerto $74,3 \%$ a $99,7 \%$, entre o sistema e a marcação manual, de acordo com o tipo de onda analisada. O presente estudo foi concebido com a intenção de ser uma ferramenta prática e por isso voltada para a aplicação clínica. Os resultados apresentados mostram uma técnica eficaz e capaz de aperfeiçoar o processo de avaliação dos PEATEs. A técnica proposta se mostra precisa mesmo na presença de ruído, característico de sinais biológicos especialmente no PEATE por ser um sinal de amplitude baixa.

Palavras-chave Potenciais evocados auditivos de tronco encefálico, Detector automático, Transformada Wavelet Contínua, Regressão linear.

\section{Decomposition and analysis of auditory brainstem response}

\begin{abstract}
Auditory Brainstem Response (ABR) results from the combination of neural activity responses in the presence of sound stimuli, detected by the cortex and characterized by peaks and valleys. They are identified by Roman numerals (I, II, III, IV, V, VI and VII). The identification of these peaks is carried out by the classical manual process of analysis, which is based on the visual/manual processing of the signals. The morphological and temporal characteristics of the signal carry relevant physiological and anatomical information regarding the auditory system. However, in this visual process of analysis some difficulties may occur, specifically, the results of the analysis may vary according to the type of protocol, settings of equipment employed, and the experience of the examiner. This makes the analysis of ABR subject to the influence of many variables that may interfere on the reliability and agreement of results obtained in distinct research centers and by different examiners. Therefore, the main propose of this study was to develop and assess a system capable of automatically detecting and classifying ABR waves, which are called Jewett waves. A relevant feature of the proposed tool is that it can learn from the experience of examiners continuously. In order to evaluate the system approximately 748 samples of ABR obtained from 11 subjects were analyzed by the automatic system. These results were compared to analyses obtained from five seasoned examiners, and they showed a high level of agreement, ranging for $74.3 \%$ to $99.7 \%$, between responses given by the system and the examiners. Thus the proposed technique is proved to be accurate even in the presence of noise, especially characteristic of the ABR that is a sign of low amplitude.
\end{abstract}

Keywords Auditory brainstem response, Automatic detection, Continuous Wavelet Transform, Linear regression.

*e-mail: khelinenaves@yahoo.com.br

Recebido: 08/06/2012 / Aceito: 227/09/2012 


\section{Introdução}

O desenvolvimento de métodos de diagnóstico computadorizados, com a finalidade de auxiliar na investigação de doenças, tem sido empregado nos últimos anos, gerando assim, uma gama de sistemas especialistas complexos, ágeis e eficientes para a saúde (Pal e Mitra, 2010). Isso possibilita a aplicação de análises avançadas resultando na melhoria da investigação, diagnostico e tratamento de doenças, e consequentemente na redução de tratamentos inadequados e, sobretudo tardios.

No âmbito da saúde auditiva, a tendência é da aplicação de medidas objetivas cuja principal finalidade é diagnosticar precocemente as perdas auditivas, principalmente em populações especiais como recémnascidos e crianças. De acordo com a Organização Mundial de Saúde (World..., 2004), 1,4 milhões de crianças no mundo têm problemas auditivos. Olusanya e cols (Garcia et al., 2011) recentemente estimaram que 855 bebês nascem todos os dias em países em desenvolvimento com perda auditiva e com pouca expectativa de serem diagnosticas. No Brasil, menos de $30 \%$ das crianças com perda auditiva são diagnosticadas até 2 anos de idade (Brasil..., 2004). E um levantamento recente apresenta uma prevalência da perda auditiva em 1,2/1000 crianças nascidas (Hanna e Maia, 2010). Os problemas causados por um diagnóstico tardio impactam em alterações no desenvolvimento cognitivo e colaboram para o atraso do desenvolvimento da linguagem, conseqüente atraso no processo de aprendizado e desenvolvimento emocional (Chomsky, 2005; Fitcha et al., 2005).

Para a pesquisa dos limiares auditivos e investigação das vias auditivas, principalmente em populações especiais, o método de análise recomendado é a avaliação dos Potenciais Evocados Auditivos de Tronco Encefálico (PEATE) (American... e Joint..., 2007; American..., 2006a; Hall, 2006b; Mason, 2004; World..., 2009). O estudo dos PEATEs é um método de diagnóstico através de medidas eletrofisiológicas e que faz parte do protocolo de avaliação das perdas auditivas. Por se tratar de um método não invasivo, possui uma ampla aplicação clínica, sendo utilizado no diagnóstico de doenças otológicas e neurológicas, em sujeitos de diversas idades (Hood, 1998; Moller, 2007; Munhoz et al., 2000; Porto et al., 2011; Sininger, 2007; Stufflebeam et al., 2012).

O processo clássico de análise dos PEATE é baseado na visualização do sinal, no qual o examinador realiza manualmente a marcação para cada pico identificado (Burkard et al., 2007; Mason, 2004, Neonatal..., 2002). Nele são identificadas, suas características morfológicas e os aspectos temporais relevantes constituídos pelas ondas de Jewett sendo que os picos dessas ondas são nomeadas por algarismos romanos (I, II, III, IV, V, VI e VII) (Hall, 2006c). Os estudos realizados por Sohmer e Feinmesser, e posteriormente por Jewett e Williston (Hall, 2006b) atribuem a onda I ao potencial de ação do nervo auditivo. Portanto, a onda I é a representação do potencial de ação da parte distal do nervo auditivo quando ele sai da cóclea e entra no canal auditivo interno. A onda II é gerada na parte proximal do nervo auditivo. Sendo as ondas I e II geradas na porção extracraniana e intracraniana do nervo auditivo respectivamente.

As ondas seguintes são geradas nos centros auditivos e recebem contribuições de outras regiões. A onda III é formada por neurônios de segunda ordem no núcleo coclear, mais precisamente na porção caudal da ponte. Os neurônios de terceira ordem formam a onda IV que é gerada no complexo olivar superior e recebe influência de múltiplos geradores. A onda $\mathrm{V}$ reflete a atividade gerada na região do leminisco lateral e a sua porção negativa está relacionada aos potenciais dos dendritos do colículo inferior (Hall, 2006b; Palmer, 2007). As ondas VI e VII são atribuídas ao colículo inferior (Wilkinson e Jiang, 2006).

Cada pico desta onda ocorre dentro de um intervalo de tempo denominado de latência que tem relação direta com a região anatômica de origem (Antonelli et al., 1987; Burkard e Don, 2007) e um atraso desta latência ou, o não aparecimento da onda, pode indicar a presença de uma doença.

Entretanto, o processo de analise manual do sinal pode conter algumas incertezas, visto que o sinal apresenta alta variabilidade morfológica, tanto entre sujeitos, quanto entre diferentes medidas de uma mesma pessoa, principalmente quando realizados em momentos diferentes (Hall, 2006a). Outra fonte de incerteza é a escolha dos critérios de análise selecionados pelos examinadores para se analisar as ondas e determinar os picos das mesmas. No qual diferentes examinadores podem utilizar critérios distintos para a classificação das ondas (Misulis, 2003) produzindo importantes variações na forma arbitraria de como é definido um pico, (Burkard et al., 2007; Hall, 2006b). O que dificulta a análise manual e pode levar a interpretações equivocadas do sinal.

Em função das dificuldades encontradas na área clínica durante o processo de identificação dos picos das ondas de Jewett, principalmente por examinadores com pouca experiência; e consequente escassez de profissionais em regiões distantes de grandes centros; da subjetividade envolvida nesse processo; da importância de se melhorar o diagnóstico de doenças auditivas tanto no que se refere ao diagnóstico 
precoce da perda auditiva quanto no diagnóstico de doenças do nervo auditivo esse trabalho se propõe a desenvolver um sistema de detecção automática das ondas de Jewett.

Para a construção do sistema foi necessário o conhecimento detalhado das variáveis envolvidas na detecção visual dos picos de PEATE permitindo assim a aplicação e construção de ferramentas com base nos processos de analise das ondas em conjunto com as características do sinal biológico.

Portanto, este trabalho tem por objetivo testar a hipótese de que existe um comportamento característico na marcação dos PEATE pelos examinadores; e que este processo pode ser aprendido por um sistema capaz de detectar automaticamente os picos das ondas de Jewett.

Esse sistema traz como características principais: a capacidade de aprendizado; que possa ser alimentado com informações pelo próprio usuário; que atenda ao perfil de análise dos picos realizada pelos examinadores, além de considerar as características distintas do sinal.

\section{Materiais e Métodos}

Os PEATEs foram coletados em onze sujeitos, sendo 5 mulheres e 6 homens, todos saudáveis, sem queixas auditivas, e com idade média de 36 anos ( 20 a 52 anos). Os participantes foram selecionados na Universidade Federal de Uberlândia e assinaram o Termo de Consentimento Livre e Esclarecido, aprovado pelo Comitê de Ética da Universidade Federal de Uberlândia sob o parecer n ${ }^{\circ}$ 249/06 e registro CEP: 160/06.

Para a seleção dos voluntários o critério de inclusão utilizado levou em consideração o de normalidade auditiva (normal de 0 a 25 dBNPS (Lloyd e Kaplan, 1978 apud Katz, 1989)), confirmada através de exames prévios de otoscopia, audiometria tonal e testes de logoaudiometria (IRF-Índice de Reconhecimento de Fala e LRF-Limiar de Recepção de Fala).

Para a coleta dos sinais foi utilizado o amplificador comercial Bio-logic's Evoked Potential System (EP), da marca Bio-Logic. Os eletrodos foram posicionados segundo o sistema internacional 10-20 (Böcker et al., 2005), nas seguintes derivações: Mastoide (M1 e M2), Referência (Cz) e Terra (Fz) (American..., 2006b). Foram utilizados os fones TDH39 para geração dos estímulos auditivos deflagrados pelo padrão sonoro conhecido como "Cliques" nas intensidades de 80, 60, 40 e 20 dBNA (Nível de Audição), sendo coletadas duas amostras em cada orelha e para cada intensidade.

Os sinais foram coletados em uma taxa de amostragem de $37.101 \mathrm{~Hz}$, sendo este padrão de amostragem disponível no equipamento utilizado neste estudo. O processo de promediação foi feito em 2000 amostras o que resulta em uma melhor relação sinal/ruído. A taxa de estimulo utilizada foi de 21 ciclos/s como recomendado na literatura (American..., 2006b; American..., 1987)

Após a coleta dos PEATEs, foram coletadas as marcações das ondas de Jewett realizadas por cinco examinadores (E1, E2, E3, E4 e E5), em 160 amostras de PEATE, que consistiu em identificar as ondas I, II, III, IV e V, nas intensidades de 80, 60, 40 e 20 dBNA, seguindo o critério individual para a orelha direita e orelha esquerda. As marcações foram utilizadas para a avaliação da variabilidade entre examinadores, bem como para a criação da base de conhecimento utilizada para o treinamento do classificador.

O sistema de detecção foi implementado utilizando-se o ambiente Matlab 7.0. Para a construção do sistema proposto, a arquitetura do modelo foi dividida em Detector de Picos e Classificador Probabilístico. A Figura 1 ilustra as etapas de desenvolvimento do sistema automático.

\section{Detector de picos}

$\mathrm{Na}$ etapa de detecção dos picos foi realizado o cálculo da Transformada Wavelet Continua (TWC) no sinal, aplicando-se os parâmetros e funções especialmente ajustados para os sinais de PEATE. A TWC é definida como a soma sobre todo o domínio temporal do sinal multiplicado por versões escalonadas e deslocadas da função Wavelet mãe $(\Psi)$, descrito na Equação 1.

$C(a, b)=\int_{-\infty}^{+\infty} f(t) \psi a, b(t) \cdot d t$

Os parâmetros $a$ e $b$ correspondem aos fatores de escala e de deslocamento, respectivamente. Para os coeficientes da função $\mathrm{C}_{\mathrm{a}, \mathrm{b}}$, onde $f(t)$ representa o sinal PEATE filtrado e $\Psi a, b(t)$ representa a wavelet mãe, foram utilizados os valores de 1 a 256 para a escala $a$ para cada ponto amostrado do sinal e a partir desses valores foram calculados os valores dos coeficientes $b$, onde $b$ varia de 1 até a extensão do sinal.

O próximo passo foi relacionar os picos das ondas de Jewett com o respectivo coeficiente e para isso foram geradas as curvas de nível a partir do gráfico da transformada. Essas curvas interceptam o eixo horizontal exatamente nos pontos de menor módulo dos coeficientes onde estão os picos e vales do sinal de PEATE. Fazendo uma relação das colunas das curvas de nível com os pontos do sinal obtiveram-se os pontos de inflexão do sinal, então se excluiu os vales. Após essa exclusão têm-se os pontos candidatos a serem atribuídos como picos de ondas de Jewett.

A aplicação da Transformada Wavelet possibilita a visualização da variação da energia do sinal em função do tempo e da freqüência (representada pelas escalas) 


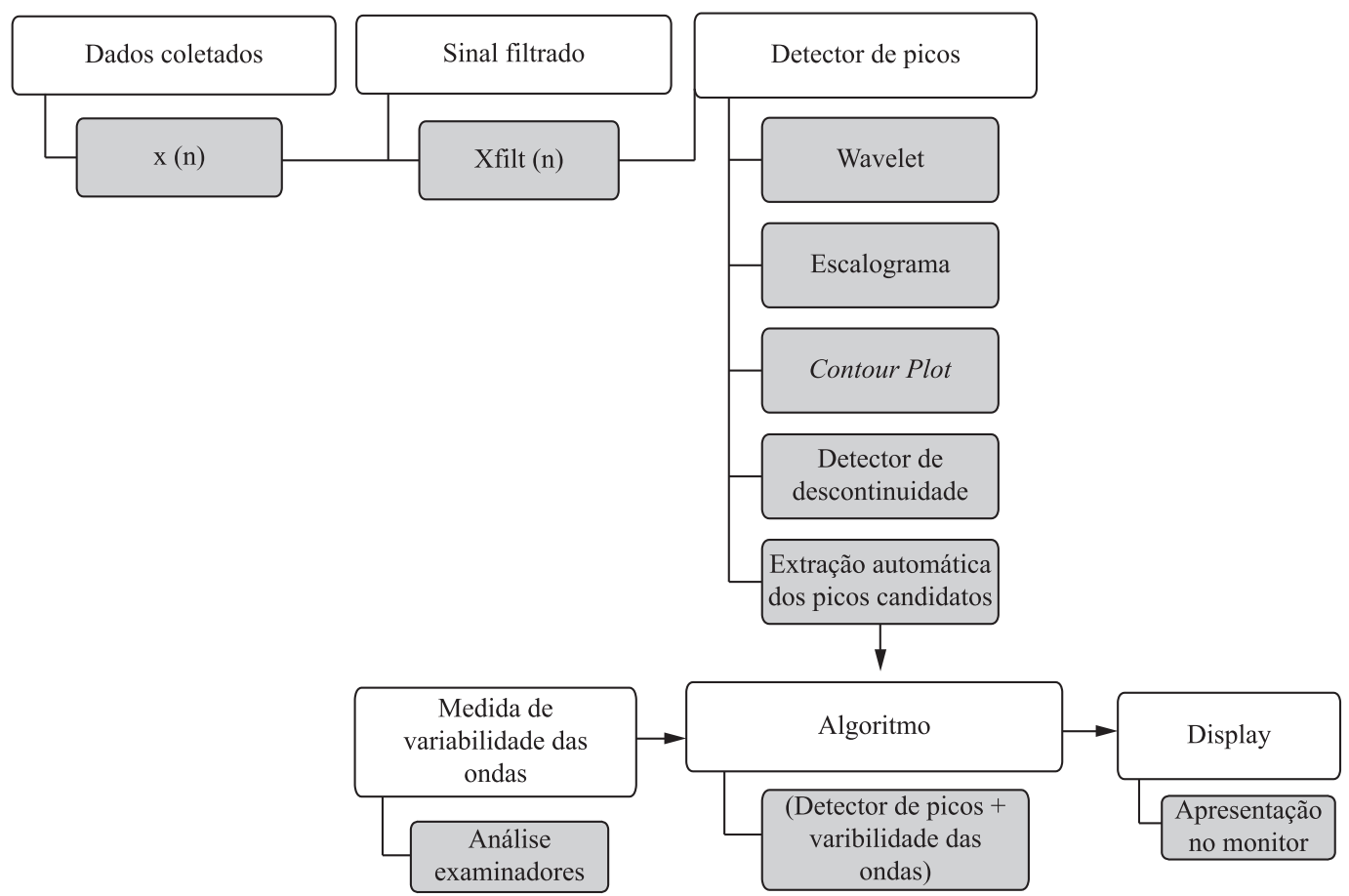

Figura 1. Diagrama de blocos representando o fluxo de dados do sistema para a detecção automática dos picos de PEATE.

Figure 1. Block diagram representing a data flow of the automatic system detection of the peaks of ABR.

em um gráfico denominado escalograma bidimensional (Moreau et al., 1999; Nenadic and Burdick, 2005; Pal e Mitra, 2010). A partir do escalograma é possível obter uma avaliação mais detalhada da energia do sinal por meio de contour plots, que representam a localização dos diferentes níveis de energia do sinal como mostra a Figura 2a.

Através das análises, observou-se que o tempo de ocorrência dos "picos candidatos" coincide com a descontinuidade encontrada no contour plots e também no escalograma. A Figura 2 ilustra este achado, no qual o PEATE é apresentando juntamente com as marcações das ondas I, II, III, IV e V, representados por círculos, realizadas por um examinador. A linha vertical incluída no gráfico mostra como a ocorrência destes picos relaciona-se com as descontinuidades no escalograma (a) e seu contour plot (c).

O resultado dessa aplicação foi um gráfico com os valores de coeficientes no tempo. Nesse gráfico é possível detectar os pontos de inflexão que são representados pelos coeficientes de menor módulo que podem ser vistos pela região mais escura no gráfico. Quanto mais escuro for o coeficiente menor será seu módulo.

Tomando como base a ocorrência das descontinuidades nos pontos em que havia possíveis picos, criou-se uma rotina de identificação das continuidades dos countour plots. Dado uma imagem de um contour plot, o programa é capaz de retornar um conjunto de pontos em que os contornos cruzaram o eixo horizontal. A partir dessa informação os pontos referentes às descontinuidades e identificados no gráfico são considerados picos candidatos.

\section{Classificador probabilístico}

Após a etapa de detecção dos picos candidatos as ondas de Jewett, foi então desenvolvida a etapa de identificação das ondas de Jewett, baseada em um modelo probabilístico a partir dos dados de marcações dos picos realizados pelos examinadores. Para a identificação dos picos candidatos o banco de dados de marcações foi dividido por ondas I, II, III, IV e V, e por intensidades de 80, 60, 40 e 20 dBNA.

A verificação da consistência dos valores de marcação foi feita com base nos valores de latências das ondas obtidas pelos examinadores, que se revelaram coerentes com os padrões encontrados na literatura. Como por exemplo, na intensidade de 80 dBNA foram: ondas I - 1,56 ms, III - 3,77 ms e V - 5,53 ms (Hall, 2006a; Hood, 1998; Misulis, 2003; Musiek et al., 2007), revelando assim a consistência dos dados na detecção visual das ondas de Jewett.

Para a analise da concordância entre os examinadores utilizou-se por meio de estatística descritiva o método de categorização dos dados em grupos distintos, tendo como base os valores do 

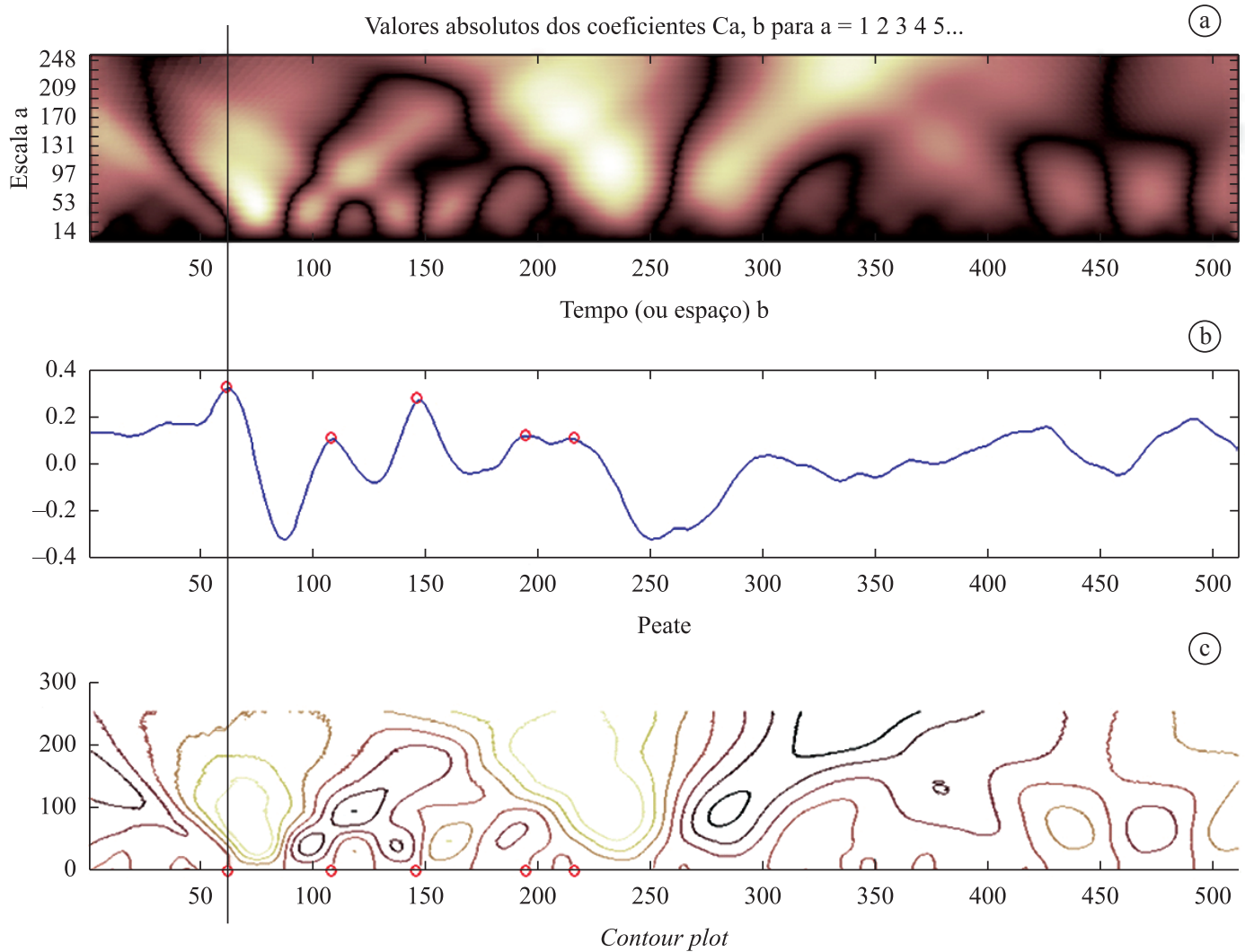

Figura 2. Escalograma na parte superior, sinal de PEATE na parte intermediária e contour plot na parte inferior. Os circulos representam as marcações dos picos realizadas pelos examinadores para este sinal.

Figure 2. Scalogram at the top, ABR signal at the middle and bottom contour plot. The circles represent the markings made by the examiners of the peaks for this signal.

padrão de reprodutibilidade sugeridos por Burkard e Don (2007), Don (1989), Hood (1998) e Vannier (2002). Essa discrepância foi categorizada em quatro grupos: nula (sem diferenças) leve ( $<0,1 \mathrm{~ms})$, moderada (entre 0,1 e 2,0 ms) e grave ( $>0,2 \mathrm{~ms})$. A frequência de ocorrência foi estimada para cada categoria.

Para cada intensidade foi estabelecido o modelo probabilístico através de um arranjo de uma distribuição Gaussiana que se caracteriza por uma distribuição normal dos dados das marcações. O resultado foi uma distribuição Gaussiana para cada tipo de onda e para cada intensidade utilizada neste trabalho.

A partir dessas distribuições calculam-se as médias e os desvios padrão das latências e das amplitudes das marcações. Usando esses parâmetros estatísticos foi possível determinar a probabilidade de um pico detectado ser o pico de uma das ondas de Jewett. E finalmente, para cada onda, os pontos que apresentarem as maiores probabilidades são definidos como picos da onda.

Para avaliar o desempenho do detector automático, foi utilizado um banco de dados teste com 588 amostras. No qual um examinador realizou a marcação dos picos em todo o banco de dados e separadamente foram realizadas as marcações dos picos pelo sistema de forma automática. Essas informações foram armazenadas em vetores de dados e em seguida, realizada a análise comparativa entre as marcações.

\section{Resultados}

Através do estudo do comportamento de marcação dos PEATEs pelos especialistas, foi possível identificar os valores de latência para cada onda e em cada intensidade, determinar os valores de concordância entre os mesmos e o modelo de analise esperado para cada onda.

Esta análise resultou em uma taxa de concordância entre os examinadores de $81,62 \%$, considerando as categorias, nula e leve. Esse número aumenta para $96,21 \%$ quando considerada a categoria moderada. Diferenças maiores que 0,2 ms, que não são aceitáveis e representam 3,79\% do total de amostras. Esses parâmetros são importantes não somente para verificar se existe uma subjetividade considerável, como também para validar o sistema automático proposto. 
O segundo passo foi o estudo da variabilidade das marcações das ondas de Jewett e pelos resultados obtidos pela Regressão Linear $\left(y=\beta_{0}+\beta_{1} x\right)$, foi possível se extrair e analisar os parâmetros do modelo para a compreensão da relação entre examinadores (Hanna e Maia, 2010; Stufflebeam et al., 2012). A Figura 3 ilustra os parâmetros as distribuições de cauda estreita encontrado para a onda V.

\section{O Sistema de marcação Automática ABEP}

$\mathrm{Na}$ Figura 4, observam-se as diferenças encontradas em porcentagem para cada onda. Observe que para a onda $\mathrm{V}$ a marcação do especialista e a marcação automática, possuem uma discrepância maior quando comparada com as demais ondas. Isto se deve em parte ao fato da onda V ser evidente mesmo em baixas intensidades onde se observa a diminuição da relação sinal/ruído, resultando em uma grande faixa de valores possíveis, o que dificulta a escolha de um ponto determinado e consequente aumento da discrepância.

$\mathrm{Na}$ Tabela 1, revela a porcentagem de acerto do sistema para cada onda e em cada intensidade avaliada. Para a intensidade de $80 \mathrm{dBNA}$ as principais componentes são as ondas I, III e V, no qual podemos verificar uma taxa de acerto de $91,9 \%$ a 94,8 . Para a pesquisa de limiares auditivos a componente mais importante do sinal é a onda $\mathrm{V}$ que permanece mesmo em intensidades baixas de estimulo e para a onda $\mathrm{V}$ temos uma taxa de acerto de $74,3 \%$ a $91,9 \%$. No contexto geral o sistema automático apresenta uma taxa de acerto global de $93,45 \%$ na marcação das ondas e em diferentes intensidades. Quando comparado com o ponto de vista clínico, ou seja, os métodos de marcação manual de um especialista, que apresenta uma taxa global de concordância de $96,21 \%$, pode se considerar que o sistema automático alcança $97,13 \%$ de eficiência. É importante considerar o comportamento de marcação manual no processo de avaliação de um sistema automático, pois o mesmo, a princípio, deve estar coerente com o método utilizado por ser o melhor parâmetro de avaliação.

$\mathrm{Na}$ Figura 4 abaixo é possível visualizar a interface construída para a detecção automática.

\section{Discussão}

Para que a construção do sistema de detecção automática das ondas de Jewett fosse alcançada, foi realizado primeiramente o estudo do comportamento de marcação das ondas bem como da variabilidade dessas marcações pelos examinadores e para tal a colaboração dos mesmos nesse processo foi de fundamental importância. Embora o número de

Onda V

E1E2

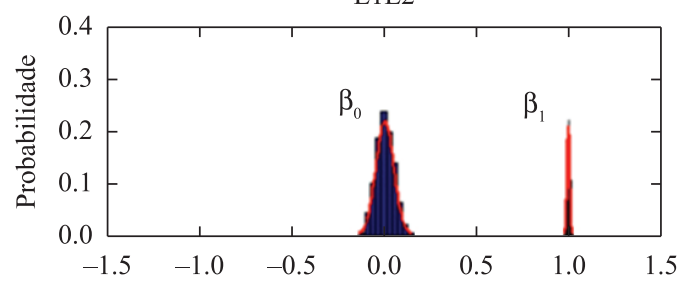

E1E4
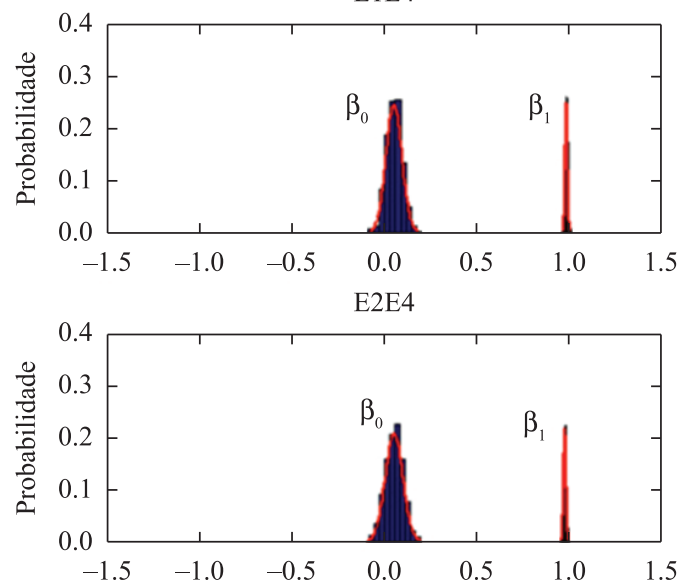

E1E3

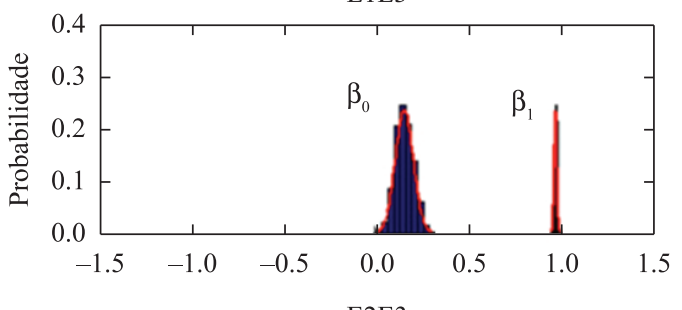

E2E3
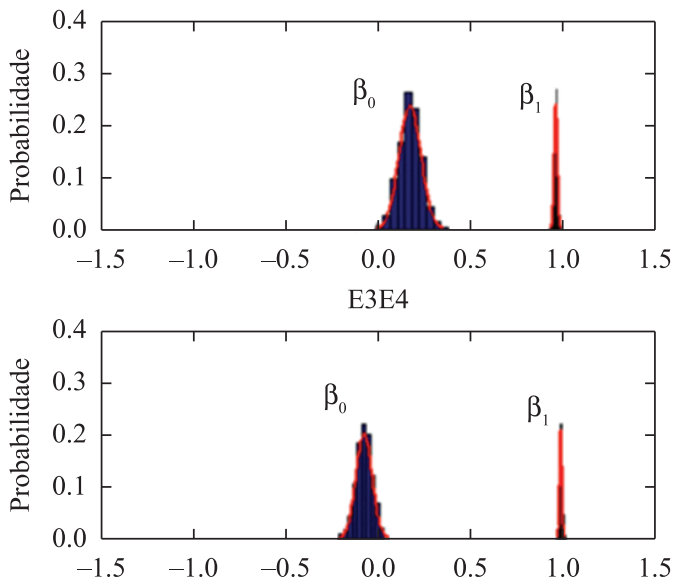

Figura 3. Probabilidade de distribuição dos parâmetros $\beta_{0}$ (esquerda) e $\beta_{1}$ (direita) para a onda V, pelos pares de examinadores. Figure 3. Probability distribution function of the parameters $\beta 0$ (left) and $\beta 1$ (right) for wave $V$, for each pairs of the examiners. 


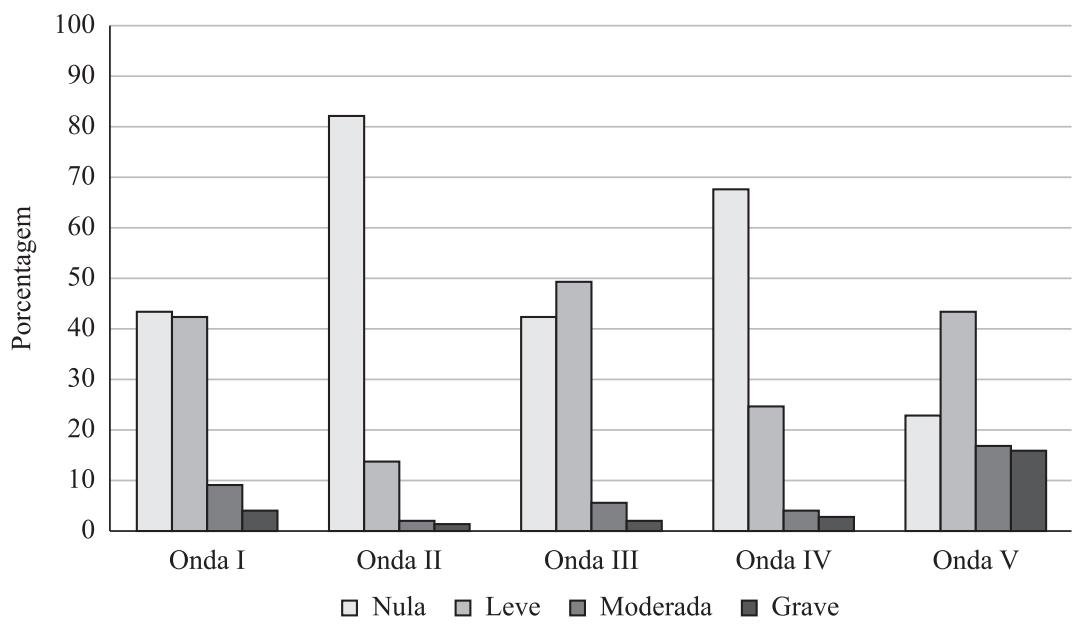

Figura 4. Gráfico das diferenças encontradas entre as marcações automáticas e as marcações manuais. Os valores foram categorizados e distribuídos para cada onda.

Figure 4. Graph of the differences found between the automatic marks and manual marks. Values were categorized and distributed for each wave.

Tabela 1. Porcentagem de acertos do sistema automático divididos por intensidade do estímulo sonoro e categorizado por onda.

Table 1. Percentage of correct automatic system divided by sound stimulus intensity and categorized by wave.

\begin{tabular}{|c|c|c|c|c|c|}
\hline Categoria & I & II & III & IV & $\mathbf{V}$ \\
\hline \multicolumn{6}{|c|}{80 dBNA } \\
\hline Nula & 17,4 & 66,8 & 22,3 & 42,1 & 20,1 \\
\hline Leve $(<0,1 \mathrm{~ms})$ & 65,1 & 26,1 & 67,6 & 44,5 & 60,5 \\
\hline Moderada $(0,1-0,2 \mathrm{~ms})$ & 12,3 & 4,4 & 5 & 6,4 & 11,3 \\
\hline Grave $(>0,2 \mathrm{~ms})$ & 5,2 & 2,7 & 5,2 & 7 & 8,1 \\
\hline Total & 100 & 100 & 100 & 100 & 100 \\
\hline \multicolumn{6}{|c|}{60 dBNA } \\
\hline Nula & 71,4 & 98,7 & 23,8 & 69,8 & 14,7 \\
\hline Leve $(<0,1 \mathrm{~ms})$ & 18,7 & 1 & 65,1 & 24,6 & 50,2 \\
\hline Moderada $(0,1-0,2 \mathrm{~ms})$ & 6,3 & 0 & 10,5 & 4,3 & 24,4 \\
\hline Grave $(>0,2 \mathrm{~ms})$ & 3,6 & 0,3 & 0,6 & 1,3 & 10,7 \\
\hline Total & 100 & 100 & 100 & 100 & 100 \\
\hline \multicolumn{6}{|c|}{40 dBNA } \\
\hline Nula & - & - & 87,40 & 96,2 & 14,9 \\
\hline Leve $(<0,1 \mathrm{~ms})$ & - & - & 10,60 & 2 & 41,7 \\
\hline Moderada $(0,1-0,2 \mathrm{~ms})$ & - & - & 1,20 & 1,4 & 21 \\
\hline Grave $(>0,2 \mathrm{~ms})$ & - & - & 0,80 & 0,4 & 22,4 \\
\hline Total & - & - & 100 & 100 & 100 \\
\hline \multicolumn{6}{|c|}{20 dBNA } \\
\hline Nula & - & - & - & - & 44,2 \\
\hline Leve $(<0,1 \mathrm{~ms})$ & - & - & - & - & 18,4 \\
\hline Moderada $(0,1-0,2 \mathrm{~ms})$ & - & - & - & - & 11,8 \\
\hline Grave $(>0,2 \mathrm{~ms})$ & - & - & - & - & 25,7 \\
\hline Total & - & - & - & - & 100 \\
\hline
\end{tabular}

examinadores que participaram neste estudo seja pequeno, dado a dificuldade de disponibilidade desses profissionais, quanto maior o número de marcações manuais contidas no banco de dados, maior será a fidelidade das marcações automáticas ao padrão estabelecido pelas marcações do banco, o que resultará em uma Função de Probabilidade de Distribuição (FPD) mais precisa.

Para compreender esse comportamento foi necessário, o estudo da melhor técnica para analisar essa variabilidade e que representasse mais fielmente esse comportamento em todas as intensidades de estimulo avaliadas e para cada onda. Em seguida foram realizados os estudos para o levantamento da técnica mais adequada para o processamento de sinais, e finalmente a forma de alimentação do modelo probabilístico. De posse de todas essas ferramentas o sistema foi construído e testado.

Os resultados obtidos pelo sistema podem ser comparados com outros estudos em detecção automática utilizando PEATE como os de Vannier (2002), com acurácia acima de $90 \%$, no estudo de Davey et al. (2007) com acurácia de 95,6\% no primeiro estágio e $85 \%$ no segundo estágio, Sanchez et al. (1995) com 90,8\% a 98,5\%, Ndit-Ali et al. (2000) com $83 \%$ foram considerados pelos autores como sendo resultados considerados satisfatórios.

Rushaidin (2009), em seu estudo utilizou a energia instantânea do sinal através da aplicação da Transformada Rápida de Fourier (FFT) e da Transformada Wavelet. Esta técnica se revelou uma boa proposta de detecção, entretanto foi avaliado o sinal apenas na intensidade de 80 dBNA e para se avaliar o desempenho no que foi proposto o mesmo deveria detectar outros sinais em diferentes níveis de intensidade.

O estudo de Davey et al. (2007), o sistema desenvolvido foi baseado em um modelo de classificação que utilizava as informações de tempo, frequência e medidas de correlação. O classificador empregou duas técnicas as Redes Neurais Artificiais e o algoritmo de decisão que eles citam como sendo o C5.0. 


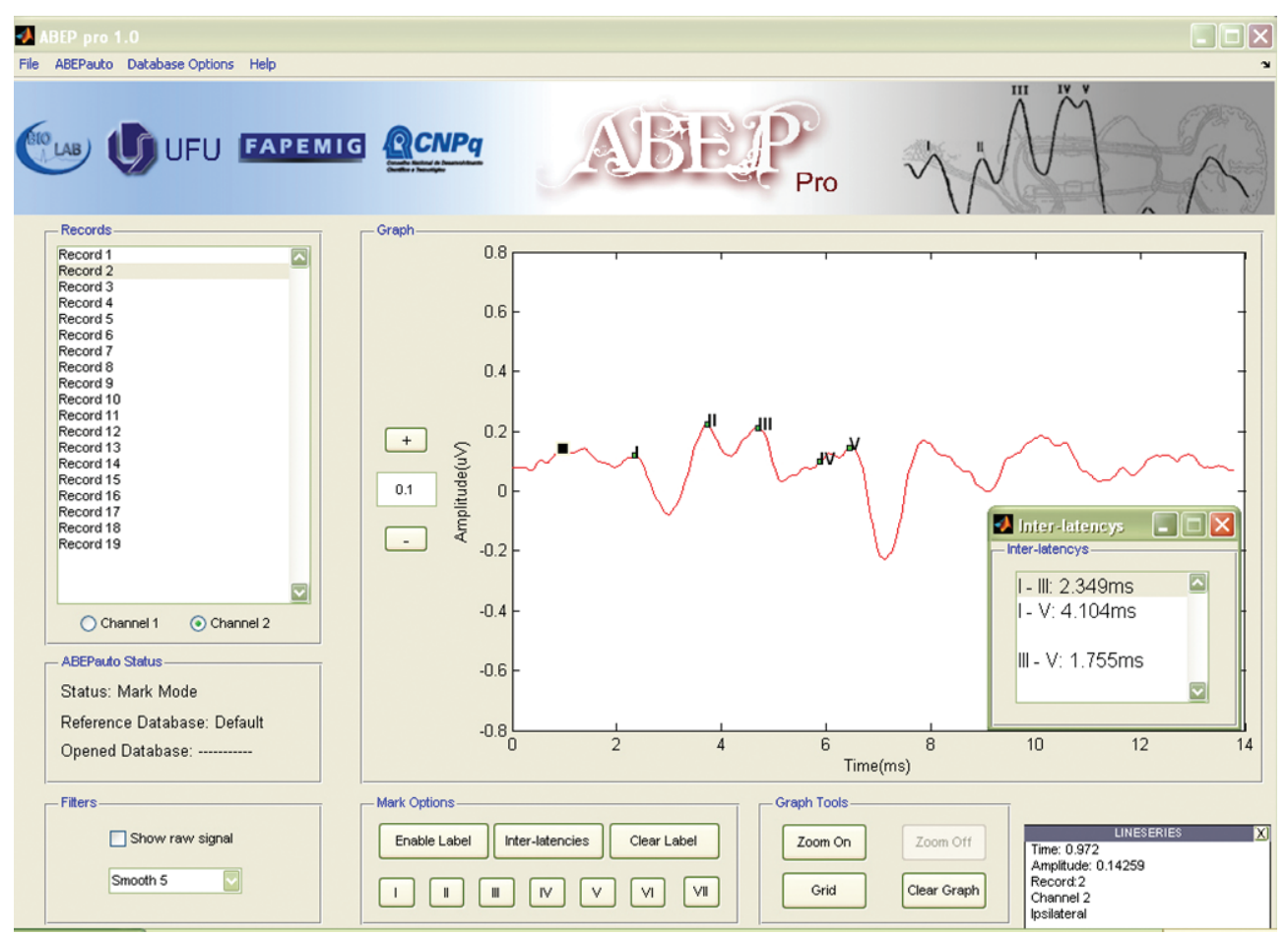

Figura 5. Interface com as gravações das ondas e o sinal de PEATE. Observe que os elementos necessários para a marcação das ondas como menu e painel de controle estão dispostos de forma amigável e intuitiva. O sinal apresentado foi marcado pelo detector automático. Utilizado filtro smooth de ordem 5. Uma janela com os valores de intervalos interpicos pode ser visualizado e abaixo outra janela com os detalhes do sinal. Figure 5. Interface with recording waves and signal ABR. Note that the necessary elements for marking waves as menu and control panel are arranged amicably and the intuitive way. The signal presented was marked by the automatic detector. Used smooth filter with $5^{\text {th }}$ order. A window with the values of interpeak intervals can be viewed below and another window with the details of the signal.

A acurácia foi validada utilizando treinamento aleatório em um conjunto de dados validados, cada onda foi classificada por um único examinador e o resultado teve dois estágios. Sendo que o primeiro utilizou a relação da energia de pré-estímulo e pós-estímulo no domínio do tempo e as medidas de potência no domínio da frequência, apresentando uma precisão de $95,6 \%$. $\mathrm{Na}$ segunda etapa, foram utilizados dados de entrada do domínio do tempo, frequência e classificador de correlação combinados com o método de DempsterSshafer que produz um modelo híbrido, revelando uma acurácia de $85 \%$.

$\mathrm{O}$ algoritmo proposto no presente estudo se diferencia dos demais em sua metodologia e se propõe a ser um método não supervisionado automático baseado na Transformada Wavelet Contínua (TWC), e em métodos probabilísticos.

Quando analisadas as técnicas utilizadas na literatura para detecção automática dos picos das ondas de Jewett, nenhum dos estudos levou em consideração a utilização de forma integrada de quatro pré-requisitos importantes: a) o perfil de marcação de ondas para um grupo de examinadores, b) o estudo da correlação entre examinadores, c) a capacidade de aprendizado do sistema e d) a qualidade do sinal coletado. Esses pré-requisitos quando utilizados em conjunto melhoram a performance do sistema automático tornando-o mais confiável.

Pelos resultados obtidos nos testes o sistema apresentou uma taxa de assertividade quando comparado a análise clássica manual de $93,45 \%$. Considerando que a taxa de concordância entre os especialistas é de $96,21 \%$, podemos concluir que o sistema apresentou bom desempenho.

O presente estudo foi concebido com a intenção de ser uma ferramenta prática e por isso voltada para a aplicação clínica. Os resultados apresentados mostram uma técnica eficaz e capaz de agilizar o processo de avaliação dos PEATE. Podendo contribuir no diagnóstico precoce das perdas auditivas, pois uma das maiores barreiras na identificação de problemas auditivos declaradas no estudo de Garcia et al. (2011) está na escassez de material humano (pessoal qualificado, como otorrinolaringologistas e fonoaudiólogos). E o sistema desenvolvido uma vez que alimentado com um banco de dados confiável pode ser manipulado por profissionais sem muita experiência técnica, possibilitando uma agilidade nos processos de avaliação auditiva. 


\section{Conclusão}

A proposta do presente trabalho foi desenvolver de um sistema automático de detecção de picos que apresenta como característica principal a capacidade de aprendizado. Isso foi possível levando-se em consideração características especificas no que diz respeito ao sinal analisado e ao processo de marcação realizado por especialistas. Os dados de marcação serviram de entrada para alimentar o sistema que, a medida os valores de picos são adicionados essa informação passa a fazer parte do classificador, sendo assim quanto mais informações de entrada são disponibilizadas no sistema mais ajustado ao perfil do examinador o sistema ficará. Poderá no futuro ser incluídos bancos de dados de centros de pesquisa ou de pesquisadores de referência na área que servirão como parâmetros durante a análise do sinal de PEATEs, bem como poderá se criar um perfil específico de analise de PEATEs em um serviço de saúde auditiva de clínicas e hospitais.

O sistema proposto contribui para que o tempo de avaliação dos PEATE possa ser reduzido, permitindo além de uma análise eficiente a otimização do tempo gasto no processo de análise. E para regiões aonde não existam examinadores especializados o sistema poderá ser manipulado por examinadores com pouca experiência servindo de auxilio nas avaliações e consequentemente na avaliação precoce dos problemas auditivos.

É importante destacar as contribuições desse estudo para a criação de um sistema automático com capacidade de aprendizado, no qual resultou primeiramente em um modelo que representa a relação entre examinadores distintos. Os parâmetros deste modelo, juntamente com sua variabilidade podem ser usados para gerar novos modelos, para gerar novos conjuntos de dados que levem em conta as diferenças subjetivas entre examinadores. Modelos genéricos como este pode ser usado para gerar os dados conhecidos, com características diferentes controladas pela variabilidade do conjunto de dados original, que pode ser empregado para avaliar sistemas desenvolvidos para a detecção automática de ondas Jewett. Outra contribuição foi demosntrar a aplicação da TWC no sinal de PEATE, através das analises dos parâmetros como a energia do sinal em função do tempo e da frequência de forma rápida em um conjunto de dados, melhorando assim o tempo de análise. Esta técnica é simples de ser aplicada e requer menos tempo de processamento quando comparada com as técnicas já utilizadas, permitindo assim a implementação em computadores portáteis podendo ser operado por profissionais da saúde. E por fim, como visto na Figura 5, a criação de um software capaz auxiliar a avaliação dos PEATEs automaticamente, possibilitando um armazenamento em tempo real das marcações em seu banco de dados e a criação de estilos para cada examinador.

\section{Agradecimentos}

Os autores agradecem a Fundação de Amparo e Pesquisa de Minas Gerais (FAPEMIG), pelo suporte financeiro nessa pesquisa (FAPEMIG - TEC APQ2481-6.01/07). E ao pesquisador Guilherme Cavalheiro pelo suporte dado neste estudo.

\section{Referências}

American Academy of Pediatrics, Joint Committee on Infant Hearing. Position statement: Principles and guidelines for early hearing: Detection and intervention programs. pediatrics. 2007;120(4):898-921. PMid:17908777. http:// dx.doi.org/10.1542/peds.2007-2333

American Clinical Neurophysiology Society. Guideline 9A: Guidelines on evoked potentials. Journal of Clinical Neurophysiology. 2006b; 23(2):125-37.

American Clinical Neurophysiology Society. Guideline 9C: Guidelines on short-latency auditory evoked potentials. Journal of Clinical Neurophysiology. 2006a; 46(3):275-86.

American Speech-Language-Hearing Association. Short latency auditory evoked potentials. ASLH; 1987. p. 1-57. Available from: www.asha.org/policy.

Antonelli AR, Bellotto R, Grandori F. Audiologic diagnosis of central versus eighth nerve and cochlear auditory impairment. Audiology. 1987; 4(26):209-26. http://dx.doi. org/10.3109/00206098709081550

Böcker KBE, Avermaete JAG, Berg-Lenssen MMC. The international 10-20 system revisited: Cartesian and spherical co-ordinates. Netherlands; 2005.

Brasil. Ministério da Saúde. Política nacional de saúde auditiva. Brasília: Secretaria de Atenção à Saúde; 2004. p. 1-11.

Burkard RF, Don M. The auditory brainstem response. In: Burkard RF, Don M, Eggermont JJ, editors. Auditory evoked potencials: basic principles and clinical applications. Baltimore: Lippincott Williams \& Wilkins; 2007. p. 229-53.

Burkard RF, Eggermont JJ, Don M. Auditory evoked potentials: Basic principles and clinical application. Baltimore: Lippincott Williams \& Wilkins; 2007.

Chomsky N. Three factors in language design. Linguistic Inquiry. 2005; 36(1):1-22. http://dx.doi. org/10.1162/0024389052993655

Davey R, McCullagh P, Lightbody G, McAllister G. Auditory brainstem response classification: A hybrid model using time and frequency features. Artificial Intelligence in Medicine. 2007; (40):1-14. PMid:16930965. http://dx.doi. org/10.1016/j.artmed.2006.07.001

Don M. Quantitative approaches for defining the quality and threshold of auditory brainstem responses. IEEE Engineering in Medicine \& Biology Society 1989; 2:761-62.

Fitcha WT, Hauserb MD, Chomsky N. The evolution of the language faculty: Clarifications and implications. 
Cognition. 2005; 97:179-210. PMid:16112662. http://dx.doi. org/10.1016/j.cognition.2005.02.005

Garcia BGD, Gaffney C, Chacon S, Gaffney M. Overview of newborn hearing screening activities in Latin America. Revista Panamericana de Salud Pública. 2011; 29(3):145-52. PMid:21484013.

Hall JW. ABR Analysis and interpretation. In: Hall JW. New Handbook of Auditory Evoked Responses. Boston: Pearson Edication, Inc.; 2006a. p. 212-57. PMid:16434768.

Hall JW. New Handbook of auditory evoked Responses. Boston: Pearson Edication, Inc.; 2006b.

Hall JW. Overview of Auditory Neurophisiology. In: Hall JW. New Handbook of Auditory Evoked Responses. Boston: Pearson Edication, Inc.; 2006c. p. 1-34.

Hanna KF, Maia RA. Triagem auditiva neonatal: incidência de deficiência auditiva neonatal sob a perspectiva da nova legislação paulista. Revista Brasileira de Saúde Materno Infantil. 2010; 10(2):257-64. http://dx.doi.org/10.1590/ S1519-38292010000200013

Hood LJ. Clinical applications of the auditory brainstem response. San Diego: Singular Publishing Group Inc.; 1998.

Katz J. Audiologia clínica. New York: Manole; 1989.

Mason SM. Evoked potentials and their clinical application. Current Anaesthesia \& Critical Care. 2004; 15:392-99. http://dx.doi.org/10.1016/j.cacc.2004.12.003

Misulis KE. Potencial evocado de Spehlmann. Rio de janeiro: Revinter Ltda.; 2003.

Moller AR. Neural generators for auditory braisntem evoked potencials. In: Burkard RF, Don M, Eggermont JJ, editors. Auditory evoked potencials: basic principles and clinical applications. Baltimore: Lippincott Williams \& Wilkins; 2007. p. 336-54. PMid:22062287.

Moreau F, Gibert D, Holschneider M, Saracco G. Identification of soucers of potential fields with the Continuous Wavelet Transform: Basic theory. Jounal of Geophysical Research. 1999; 104(B3):5003-13. http:// dx.doi.org/10.1029/1998JB900106

Munhoz MSL, Silva MLG, Caovilla HH, Ganança MM, Frazza MM. Neuroanatomofisiologia da audição. In: Munhoz MSL, Caovilla HH, Silva MLG, Ganança MM. Audiologia Clinica. Sao Paulo: Athena; 2000. p. 284.

Musiek FE, Shinn JB, Jirsa RE. The auditory brainstem response. In: Burkard RF, Don M, Eggermont JJ, editors. Auditory evoked potencials: basic principles and clinical applications. Baltimore: Lippincott Williams \& Wilkins; 2007. p. 291-312.

Ndit-Ali A, Adam O, Motsch JF. Modelling and recognition of brainstem auditory evoked potentials using Symlet wavelet. ITBM-RBM. 2000; 21(3):150-57. http://dx.doi. org/10.1016/S1297-9562(00)90029-9
Nenadic Z, Burdick JW. Spike detection using the Continuous Wavelet Transform. IEEE Transactions on Biomedical Engineering. 2005; 52(1):74-87. PMid:15651566. http:// dx.doi.org/10.1109/TBME.2004.839800

Neonatal Hearing Screening and Assessment - NHSA. Click auditory brainstem response testing in babies. A recommended test protocol. NHSA; 2002. p. 1-9.

World Health Organization. Guildelines for hearing aids and services for developing coutries. World Health Organization Prevention of Blindness and Deafness; 2004.

Pal S, Mitra M. Detection of ECG characteristic points using Multiresolution Wavelet Analysis based Selective Coefficient Method. Measurement Science Review. 2010; 43:255-61.

Palmer AR. Anatomy and physiology of the auditory braistem. In: Burkard RF, Don M, Eggermont JJ, editors. Auditory evoked potencials: basic principles and clinical applications. Baltimore: Lippincott Williams \& Wilkins; 2007. p. 199-229.

Porto MADA, Azevedo MF, Gil D. Auditory evoked potentials in premature and full-term infants. Brazilian Journal of Otorhinolaryngology. 2011; 77(5):622-7. http:// dx.doi.org/10.1590/S1808-86942011000500015

Rushaidin MM, Salleh S-H, Swee TT, Najeb JM, Arooj A. Wave $\mathrm{V}$ detection using instantaneous energy of auditory brainstem response signal. American Journal of Applied Sciences. 2009; 6(9):1669-74. http://dx.doi.org/10.3844/ ajassp.2009.1669.1674

Sanchez R, Riquenes A, Pérez-Abalo M. Automatic detection of auditory brainstem responses using feature vectors. International Journa of Medical Informatics. 1995;39(3): 287-97.

Sininger YS. Source analysis of auditory evoked potentials and Fields. In: Burkard RF, Eggermont JJ, Don M, editors. The use of Auditory Brainstem Response in Screening for Hearing Loss and Audiometric Threshold Prediction. Baltimore: Lippincott Williams \& Wilkins; 2007. p. 254-74.

Stufflebeam SM, Levine RA, Gardner JC, Barbara FC, Furst M, Rosen BR. Objective detection and localization of multiple sclerosis lesions on magnetic resonance brainstem images: Validation with auditory evoked potentials. Journal of Basic \& Clinical Physiology \& Pharmacology. 2012; 11(3):231-58.

Vannier E, Adam O, Motsch J-F. Objective detection of brainstem auditory evoked potentials with a priori information from higher presentation levels. Artificial Intelligence in Medicine. 2002; 25:283-301. http://dx.doi.org/10.1016/ S0933-3657(02)00029-5

Wilkinson AR, Jiang ZD. Brainstem auditory evoked response in neonatal neurology. Seminars in Fetal \& Neonatal Medicine. 2006; 11(6):444-51. PMid:17015048. http://dx.doi.org/10.1016/j.siny.2006.07.005

World Health Organization - WHO. Newborn and infant hearing screening: Current issues and guiding principles for action. WHO Library; 2009.

\section{Autores}

\section{Kheline Fernandes Peres Naves*, Adriano Alves Pereira, Adriano Oliveira Andrade}

Laboratório de Engenharia Biomédica, Faculdade de Engenharia Elétrica, Universidade Federal de Uberlândia - UFU, Av. João Naves de Ávila, 2160, Bloco 3N, Campus Santa Mônica, CEP 38400-902, Uberlândia, MG, Brasil. 